ESAIM: PROCEEDINGS, October 2007, Vol. 22, 132-139

Gabriel Caloz \& Monique Dauge, Editors

\title{
PROCEEDINGS IN FLOW MODELLING IN THE SURROUNDINGS OF THE END PART OF A TRAWL
}

\author{
GÉraldine Pichot ${ }^{1}$
}

\begin{abstract}
This paper is devoted to the presentation of modelling procedures and of numerical simulations of the flow in the surroundings of the end part of a trawl, where fish are caught. We present first the hydrodynamical data collected in November 2004 at the IFREMER tank of Boulogne-surMer around and inside an axisymmetric rigid net model. Then we show the use of averaged NavierStokes/Brinkman equations with an eddy viscosity coupled with an equation for the turbulent kinetic energy (TKE) (RANS type model of order one) as for a turbulent fluid model. Finally, we test this last model by comparing the numerical results obtained with the code that we have written in Freefem ++ and the experimental data available.

Résumé. Cet article présente les avancées concernant la modélisation et la simulation numérique de l'écoulement autour de la poche d'un chalut. Nous présentons tout d'abord la campagne expérimentale menée en Novembre 2004 au bassin d'essais de l'IFREMER de Boulogne-sur-Mer afin de collecter des données hydrodynamiques sur l'écoulement autour d'une poche de chalut rigide axisymétrique. Nous exposons dans une deuxième partie le modèle fluide utilisé, basé sur les équations de NavierStokes/Brinkman avec viscosité turbulente couplées à un modèle de turbulence à une équation. Enfin, nous testons la capacité du modèle à reproduire l'écoulement dans le cas simple de la maquette de Boulogne-sur-Mer par l'intermédiaire du code de calcul que nous avons écrit pour le logiciel Freefem ++ .
\end{abstract}

\section{INTRODUCTION}

This study aims at describing the proceedings in flow modelling around and inside a cod-end net, which is a complex 3D structure. One has to deal with three difficulties: the complex geometry of the fluid domain, the turbulent feature of the flow and the boundary conditions at the output of the computational box.

Firstly, one has to process the complexity of the fluid domain in the surrounding of the net and the catch. Using a classical finite elements method would require a far too complex $3 \mathrm{D}$ mesh generation. It is wise to use an equivalent geometry to represent the net. An equivalent membrane is considered in [12]. However, this model is axisymmetric and its generalization to the $3 \mathrm{D}$ case seems difficult. We propose here to keep the idea of accounting for the net by the means of a porous membrane. However, instead of applying transfer equations at the membrane location, the membrane, as well as the catch considered as impermeable, are introduced continuously in the fluid equations by the means of a penalization term, based on the Brinkman law. It allows to set the same fluid equations in the entire domain, provided a permeability function is defined. Similar techniques are described and applied in different contexts (see [11], [9], [2], [1], [4], [6]). In our case, a high

${ }^{1}$ IFREMER, Technopôle Brest-Iroise, BP 70, 29280 Plouzane Cedex et IRMAR, Campus de Beaulieu, 35042 Rennes Cedex, geraldine.pichot@ifremer.fr

(C) EDP Sciences, SMAI 2007 
constant permeability value is set in the fluid domain, a low one in the catch domain. In the membrane, a permeability function has to be estimated. One can refer to section 3 for an example of such a function.

Secondly, the turbulent feature of the flow makes it difficult to perform Direct Numerical Simulation since it would require too high computer resources to deal with all various scales of turbulence. In the case of the study of the net model of Boulogne-sur-Mer, the Reynolds number is equal to $10^{5}$. That is why a Reynolds Averaged Navier-Stokes/Brinkman model with an eddy viscosity coupled to an equation for the turbulent kinetic energy (TKE) is used and averaged quantities are studied.

Thirdly, one has to define outer boundary conditions. The computational box should not disturb the flow, that is why ghost boundary conditions are derived (see [8]) and they are essential whenever the flow is turbulent at the output to avoid eddy reflections. One can see in section 3 that a classical output boundary condition is actually used in the simulations, since the outflow is laminar.

First, we present the experimental data available, collected by G. Germain and his IFREMER team of Boulogne-sur-Mer with the participation of the author of the present paper. Then we give the fluid system of equations, that is the averaged Navier-Stokes/Brinkman equations with an eddy viscosity, coupled with one equation for the TKE. Finally we conclude by describing our Freefem ++ code and by a comparison between experimental and numerical results.

\section{EXPERIMENTAL WORK}

\subsection{Experimental framework}

Experiments have been carried out in November 2004 at the IFREMER center of Boulogne-Sur-Mer (France). Velocity profiles have been measured inside and around a rigid resin made model built by the Boulogne-Sur-Mer IFREMER team (Figure 1). This model is like an axisymmetric rigid $1 / 6$ scaled cod-end net towed at a velocity about $1.25 \mathrm{~m} / \mathrm{s}$ with diamond-shaped meshes. The end of the net is filled with a resin mass modelling a catch of $30 \mathrm{~kg}$ of fishes.

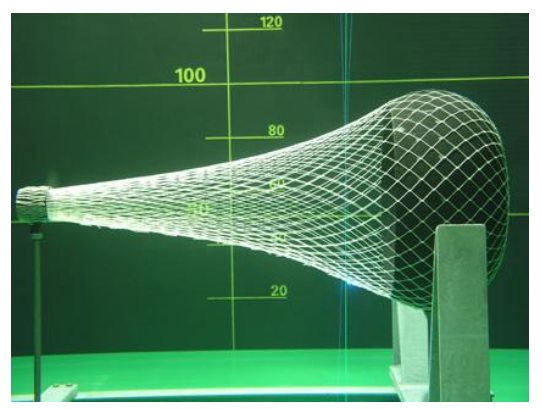

FIGURE 1. Model of the cod-end net model built at IFREMER - Boulogne-sur-Mer

Working on a rigid axisymmetric net restricts the study but it makes it easier to perform velocity measurements. The model is $1 \mathrm{~m}$ long and has an outer maximal diameter of $0.45 \mathrm{~m}$. It is maintained with a frame and set at the bottom of the IFREMER tank. The estimation of the velocity to apply in the tank comes from a Froude similitude yielding a reference velocity of $0.51 \mathrm{~m} / \mathrm{s}$.

\subsection{Experimental data}

Hydrodynamical measures are obtained using a Laser Doppler Velocimeter (LDV) technique, giving the velocity profiles at different positions, as shown on figure 2 . 


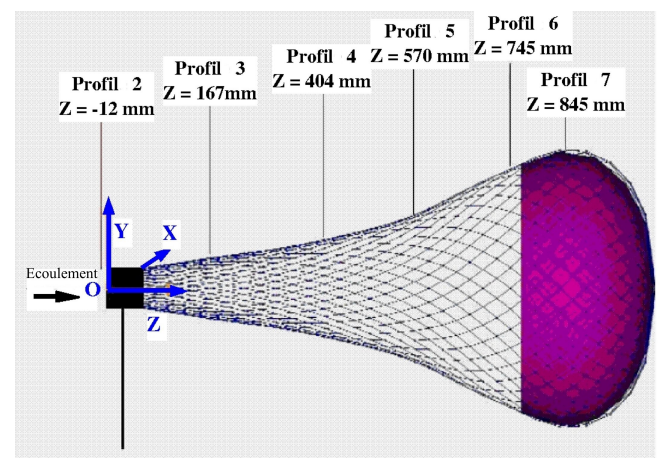

FIGURE 2. Profiles considered for the LDV measurements

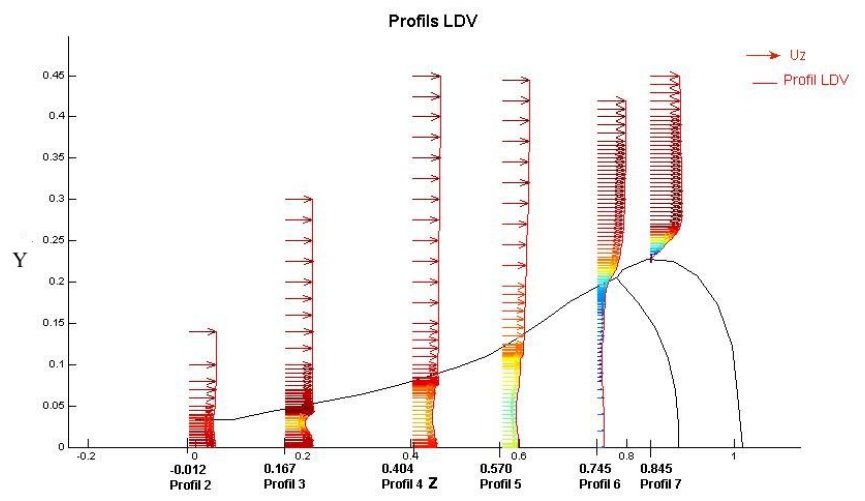

FiguRE 3. LDV profiles

One defines a cartesian coordinate system in the tank, the origin being set at the entrance of the net. We study the $\mathrm{z}$ velocity component since it has the direction of the entrance flow, and then the higher values. The results obtained for this component are displayed on figure 3 .

Our aim is now to present the turbulent model chosen to simulate the flow inside and around a rigid net, to show the results obtained with our code written in Freefem $++[5]$ and to compare them with the experimental profiles.

\section{A Reynolds Averaged Navier-Stokes/Brinkman model}

The flow around the end part of a trawl is governed by Navier-Stokes equations. Due to the presence of the catch, the twines and the nodes, the flow is turbulent. Taking the maximal catch diameter, equal to $0.45 \mathrm{~m}$, as a reference length and the entrance velocity, equal to $0.51 \mathrm{~m} / \mathrm{s}$, as a reference velocity gives a high Reynolds number equal to $10^{5}$. Therefore direct numerical simulation is compromised and a turbulent model is required (see e.g. [10]). We chose to use averaged Navier-Stokes equations with an eddy viscosity model coupled to an equation for the TKE (see [7] and [10] for generalities concerning those models), leading us to work with averaged quantities (averaged velocity and averaged pressure).

Another problem comes with the complex geometry of the fluid domain that makes any mesh generation an impossible task. The idea to overcome the difficulty of the netting is to define an equivalent net model. Let us consider the net as a porous membrane of thickness given by the minima of $\mathrm{z}$ velocity along the LDV profiles (Figure 3). 


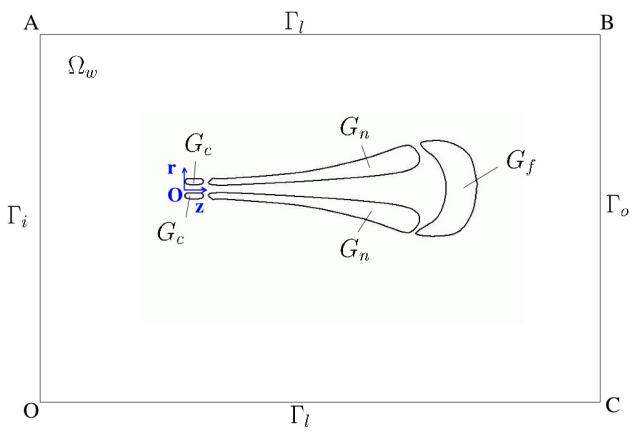

Figure 4. An equivalent net model

For a sake of simplicity, we consider in the following the axisymmetric case of the Boulogne-sur-Mer's model. The real flow is actually 3D but since we are dealing with mean quantities, the hypothesis of an axisymmetric flow around the axisymmetric cod-end net is reasonable. Then working in cylindrical coordinates allows us to reduce our $3 \mathrm{D}$ problem to the study of two components. We denote $G_{n}$ the net domain, $G_{f}$ the catch, $\Omega_{w}$ the fluid domain and $G_{c}$ the rigid frame that maintains the model in the tank (Figure 4).

It remains to determine how the porous media are taken into account in the fluid equations. This comes with a penalization method that leads to add a penalization term of the velocity in the classical Navier-Stokes equations in order to account for the presence of the net and the catch. The resulting equations are coupled with the equation for the TKE by the means of an eddy viscosity, denoted $\nu_{t}$ in the following, leading to a Reynolds Averaged Navier-Stokes/Brinkman model.

In the following we denote $(\mathbf{u}-P)$ the mean velocity and modified pressure unknowns and $k$ the TKE. The flow is governed by:

with

$$
\left\{\begin{array}{l}
\frac{\partial \mathbf{u}}{\partial t}+(\mathbf{u} \nabla) \mathbf{u}-\nabla \cdot \sigma_{\mathbf{t}}(\mathbf{u}, P, k)+\frac{1}{K(\mathbf{x})} \mathbf{u}=0, \nabla \cdot \mathbf{u}=0 \\
\sigma_{\mathbf{t}}(\mathbf{u}, P, k)=-P I d+\left(\nu_{0}+\nu_{t}\right)\left(\nabla \mathbf{u}+(\nabla \mathbf{u})^{t}\right) \\
\frac{\partial k}{\partial t}+(\mathbf{u} \nabla) k=\nabla \cdot\left(\tilde{\nu}_{t} \nabla k\right)+\frac{\nu_{t}}{2}\left|\nabla \mathbf{u}+(\nabla \mathbf{u})^{t}\right|^{2}-C_{3} \frac{k^{\frac{3}{2}}}{\ell(x)}
\end{array}\right.
$$

$$
\begin{array}{ll}
\sigma_{\mathbf{t}}(\mathbf{u}, P, k)= & -P I d+\left(\nu_{0}+\nu_{t}\right)\left(\nabla \mathbf{u}+(\nabla \mathbf{u})^{t}\right), \text { the turbulent strain tensor } \\
P & =p+\frac{2}{3} k, \text { the modified pressure } \\
= & C_{1} \ell(\mathbf{x}) k^{\frac{1}{2}}, \text { the eddy viscosity coefficient, } \\
\nu_{t} & \text { the mixing length, } \\
\ell(\mathbf{x}) & C_{2} \ell(\mathbf{x}) k^{\frac{1}{2}}, \nu_{0} \text { the kinematic viscosity of water, } \\
\tilde{\nu}_{t} & \text { adimentionalized constants. }
\end{array}
$$

The parameter $K(\mathbf{x})$ characterizes the permeability of the different media.

$$
\begin{array}{llll}
K(\mathbf{x})=\frac{1}{\epsilon} & \rightarrow & +\infty & \text { if } x \in \Omega_{w} \\
K(\mathbf{x})=\epsilon & \rightarrow \quad 0 & \text { if } x \in G_{f} \cup G_{c} \\
K(\mathbf{x})=K_{f} & & & \text { if } x \in G_{n}
\end{array}
$$


Initial and boundary conditions are the following:

$$
\left\{\begin{array}{l}
\forall \mathbf{x} \in \Omega, \quad \mathbf{u}(0, \mathbf{x})=\mathbf{u}_{0}(\mathbf{x}), \quad k(0, \mathbf{x})=k_{0}(\mathbf{x}) \\
\left.\mathbf{u}\right|_{\Gamma_{i}}=\mathbf{u}_{\mathrm{I}}=\left(u_{\mathrm{I}}, 0\right),\left.\quad k\right|_{\Gamma_{i}}=0, \\
\left.\mathbf{u}\right|_{\Gamma_{l}}=\mathbf{0},\left.\quad k\right|_{\Gamma_{l}}=0, \\
\left.\boldsymbol{\sigma}_{\mathbf{t}}(\mathbf{u}, p, k) \cdot \mathbf{n}\right|_{\Gamma_{o}}=-\frac{1}{2}(\mathbf{u} \cdot \mathbf{n})^{-}\left(\mathbf{u}-\mathbf{u}_{\mathrm{I}}\right)+(\mathbf{u} . \mathbf{n}) \mathbf{u}_{\mathrm{I}},\left.\quad \tilde{\nu}_{t} \frac{\partial k}{\partial \mathbf{n}}\right|_{\Gamma_{o}}=-(\mathbf{u} \cdot \mathbf{n})^{-} k .
\end{array}\right.
$$

In pratice, $\mathbf{u}_{0}(\mathbf{x})$ is given by solving a Stokes problem and $k_{0}(\mathbf{x})$ is set to be a constant in the entire domain. The boundary conditions at the interface $\Gamma_{o}$ are studied in [8]. It generalizes to the turbulent case the work performed in [3]. These output boundary conditions aim to avoid numerical vortices reflections at $\Gamma_{o}$ in the case the fluid would be turbulent there. Also, the existence of a solution in the sense of the distributions to our coupled problem (1)-(4) that satisfies the standard energy balance has been proved in the $2 \mathrm{D}$ case, when the eddy viscosity is bounded [8].

\section{Test of The MOdel in A SIMPle CASE}

We have tested our model to the case of the flow around the Boulogne-sur-Mer's model. Numerical simulations have been performed using the free software Freefem $++[5]$. We have decomposed the net domain $G_{n}$ in three subdomains to take the increase of permeability due to the mesh opening into account (Figure 5).

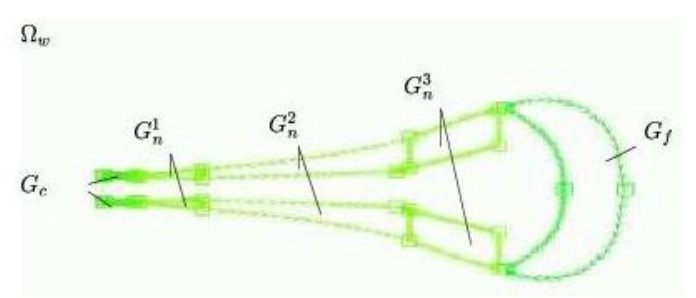

FiguRE 5. Net domain decomposition

The flow has been computed using the finite elements method. An implicit scheme is chosen for the NavierStokes/Brinkman problem and a semi-implicit one for the turbulent kinetic energy problem. Then the problems are solved iteratively until a chosen time is reached. Actually one shows that a stationary state is reached which is in agreement with the study of mean quantities. Moreover experiments suggest that the flow is laminar far from the net reducing the boundary conditions (4) to classical output boundary conditions, the ones used in the Freefem++ code:

$$
\left.\boldsymbol{\sigma}_{\mathbf{t}}(\mathbf{u}, p, k) \cdot \mathbf{n}\right|_{\Gamma_{o}}=0,\left.\quad \tilde{\nu}_{t} \frac{\partial k}{\partial \mathbf{n}}\right|_{\Gamma_{o}}=0
$$

\subsection{Numerical simulations}

An unstructured body fitted mesh is built, even in the porous and solid media (Figure 6).

After a set of simulations, one gets the value of the different parameters included in the model:

- $K_{\Omega_{w}}=10000, K_{G_{f}}=0.000001, K_{G_{c}}=0.000001, K_{G_{n}^{1}}=1, K_{G_{n}^{2}}=5, K_{G_{n}^{3}}=6$,

- Mesh: 10978 vertices; 21862 triangles,

- Time step: $0.66667 \mathrm{~s}$,

- $\ell(x)$ defined locally on each triangle as its higher side length,

- $C_{1}=0.1 ; C_{2}=0.05 ; C_{3}=0.03$,

- Thickness of the net: given by the minima of $u_{z}$ given by the LDV profiles. 


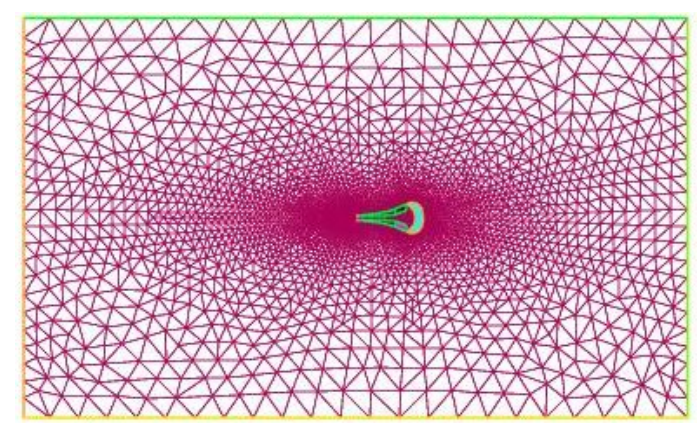

FIGURE 6. Unstructured mesh domain (10978 vertices - 21862 triangles)

Notice the permeability parameter is defined constant by parts, its values being set so as to fit the available experimental data. A matrix form may be more appropriate to control the permeability in the different directions but it brings even more parameters to set, that is why we chose a simple form in a first approximation. To find the constant values, several simulations were performed. The obtained results were consistent with what could be expected : an increase in the permeability constant in a given region increases the corresponding velocity, a decrease leads to a slowdown of the velocity. A best choice is to find the permeability in the net domain as a function of the net characteristics (like the mesh opening, the mesh orientation in the flow, ...). This is the aim of our current work.

Remark also that the length scale $\ell(\mathbf{x})$ depends on the local mesh side. As this parameter is devoted to control the turbulent kinetic energy, it mainly acts behind the catch where the turbulence rate is the highest. In comparison, the turbulence in the surroundings of the net is low. Then, a mesh refinement leads to similar velocity profiles, however it decreases the turbulent kinetic energy behind the catch. The subject of our forthcoming work is to add an equation for the length scale (leading to a $k-\ell$ model) that would prevent this mesh dependency and lead to suitable values for the turbulent kinetic energy behind the catch by comparison with experimental data.

\subsection{Comparison with experimental data}
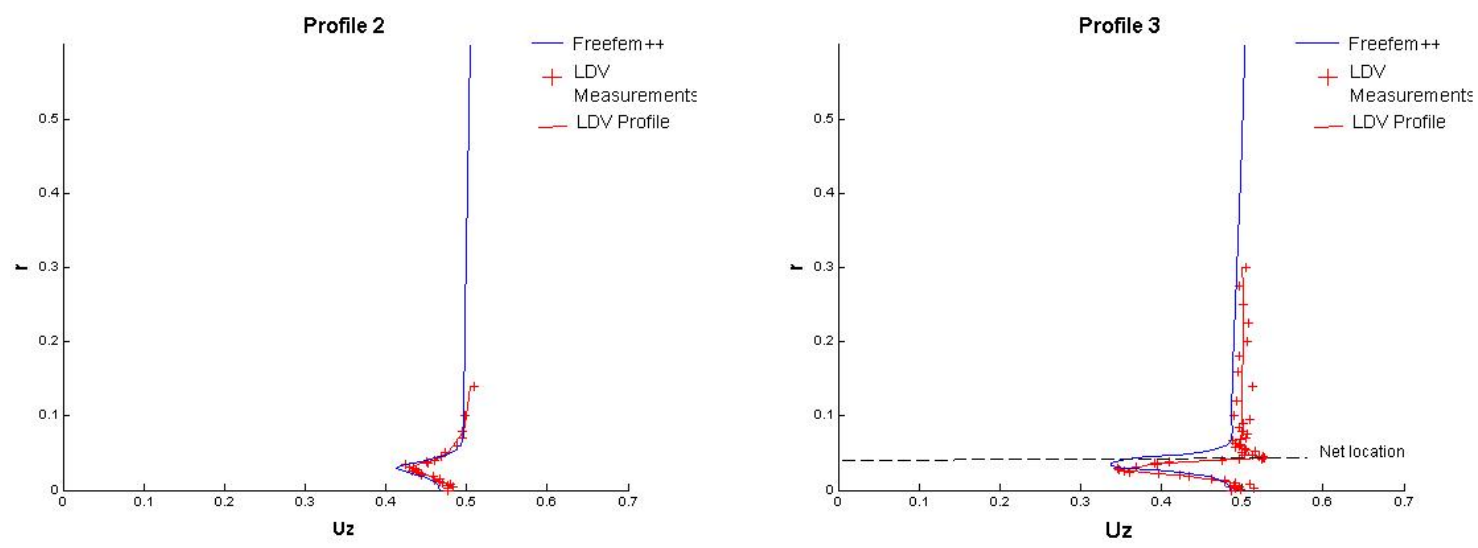

Figure 7. Profiles 2 and 3 

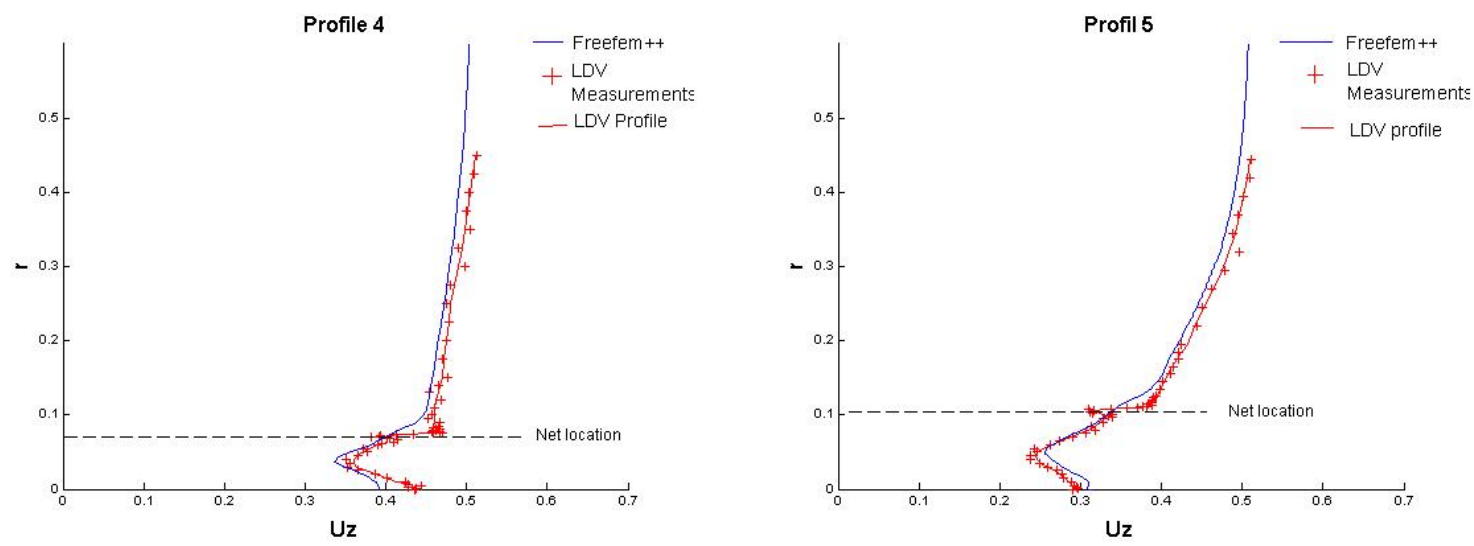

Figure 8. Profiles 4 and 5
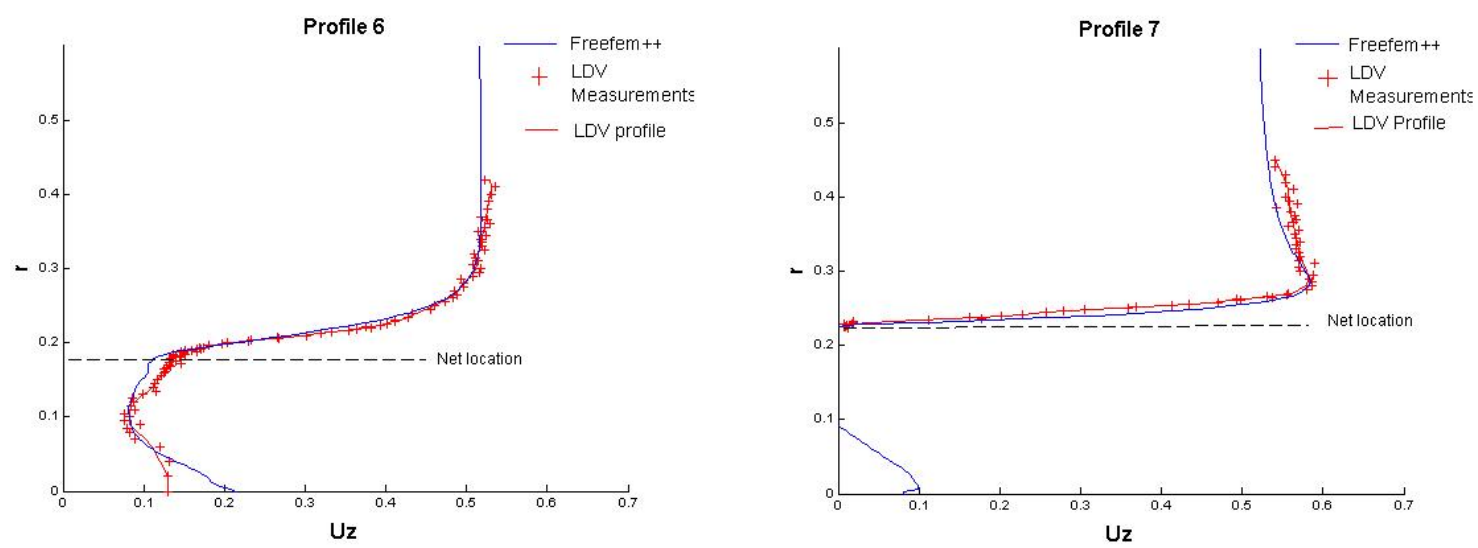

Figure 9. Profiles 6 and 7

We have compared the profiles obtained numerically and the LDV profiles (see figures 7,8 and 9 ). The results obtained with the model fit very well with the experimental data. Moreover a stationary state is reached. This is emphasized by computing the $L^{2}$ norm of the difference between two successive iterations of the velocity on one hand and of the TKE on the other hand. One observes that the difference tends to zero in both cases. Drawing the streamlines shows two expected vortices behind the catch (Figure 10).

\section{Conclusion}

The fluid model we consider is promising. The test done in the particular case of the flow around the rigid net model shows a good agreement between numerical and experimental data. The generalization of this method to the $3 \mathrm{D}$ case is in progress. More experimental data have been collected to complete the validation of the code. This will be the subject of our forthcoming work as well as the research of laws that govern the parameters required in the model (mainly the permeability, the thickness of the membrane and the length scale).

Acknowledgments. I thank IFREMER and the region Bretagne for the financial support of this work. I am very grateful toward R. Lewandowski (IRMAR, Rennes) for his relevant collaboration and I wish to express my gratitude to F. Hecht (Laboratoire J. L. Lions, Paris 6) for fruitful discussions about the numerical simulations. I also thank the IFREMER team of Boulogne-sur-Mer for making me familiar with some experimental techniques 


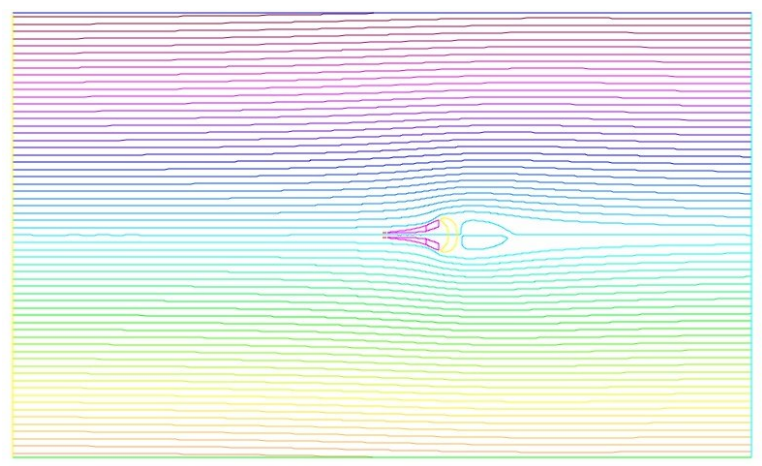

FiguRE 10. Streamlines

and for providing the experimental data. Finally I express my gratitude to D. Priour (IFREMER, Brest) for his support on this work.

\section{REFERENCES}

[1] P. Angot, C. Bruneau, and P. Fabrie, A penalization method to take into account obstacles in viscous flows., Numer. Math., 81 (1999), pp. 497-520.

[2] E. Arquis and J. P. Caltagirone, Sur les conditions hydrodynamiques au voisinage d'une interface milieu fluide-milieu poreux: application à la convection naturelle, Comptes Rendus de l'Académie des Sciences, Paris., 299(Série II) (1984), pp. 14.

[3] C. H. Bruneau And P. FAbRie, New efficient boundary conditions for incompressible navier-stokes equations: a well posedness result, RAIRO Mathematical modelling and numerical analysis, 30 (1996), pp. 815-840.

[4] G. CARbou And P. FABrie, Boundary layer for a penalization method for viscous incompressible flow, Advances in Differential equations, 8 (2003), pp. 1453-1480.

[5] F. Hecht, O. Pironneau, A. Le Hyaric, and K. Ohtsua, FreeFem ++ Manual, Laboratoire Jacques Louis Lions, Paris (http://freefem.org/ff++), 2006.

[6] K. Khadra, P. Angot, S. Parneix, and J. Caltagirone, Fictitious domain approach for numerical modelling of navierstokes equations, International journal for numerical methods in fluids, 34 (2000), pp. 651-684.

[7] R. Lewandowski, The mathematical analysis of the coupling of a turbulent kinetic energy equation to the Navier-Stokes equation with an eddy viscosity, Nonlinear Analysis TMA, 28 (1997), pp. 393-417.

[8] R. Lewandowski and G. Pichot, Numerical simulation of water flow around a rigid fishing net, Submitted, (2006).

[9] R. Mittal and G. Iaccarino, Immersed boundary methods, Annual Rev. Fluid Mech., 37 (2005), pp. $239-261$.

[10] B. Mohammadi and O. Pironneau, Analysis of the k-epsilon turbulence model, Springer, masson ed., 1994.

[11] C. S. PESkin, Flow patterns around heart valves: a digital computer method for solving the equations of motion, PhD thesis, Albert Einstein College of Medicine, 1972.

[12] B. Vincent, Etude numérique et expérimentale des écoulements guidés par une paroi perméable axisymétrique. Application à la modélisation des chaluts pour en améliorer la sélectivité, Thèse Ecole Centrale Nantes, 1996. 